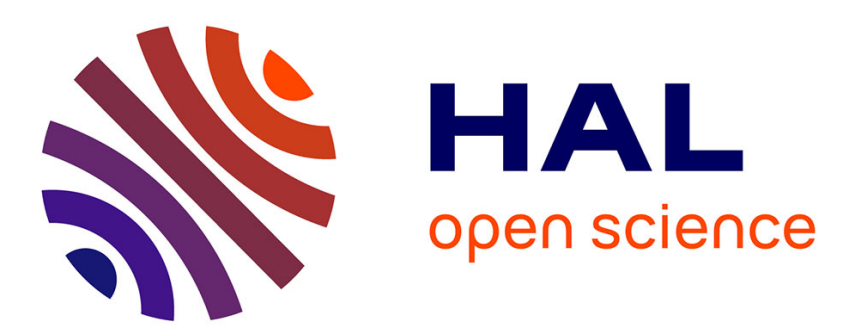

\title{
Microwave phase noise properties of optical links involving small signal and gain saturated optical amplifiers
}

Vincent Auroux, Arnaud Fernandez, Olivier Llopis, Pierre-Henri Merrer, A. Vouzelaud

\section{To cite this version:}

Vincent Auroux, Arnaud Fernandez, Olivier Llopis, Pierre-Henri Merrer, A. Vouzelaud. Microwave phase noise properties of optical links involving small signal and gain saturated optical amplifiers. 28th European Frequency and Time Forum - EFTF 2014, Jun 2014, Neuchâtel, Switzerland. pp.NC. hal-01024813

\section{HAL Id: hal-01024813 https://hal.science/hal-01024813}

Submitted on 16 Jul 2014

HAL is a multi-disciplinary open access archive for the deposit and dissemination of scientific research documents, whether they are published or not. The documents may come from teaching and research institutions in France or abroad, or from public or private research centers.
L'archive ouverte pluridisciplinaire $\mathbf{H A L}$, est destinée au dépôt et à la diffusion de documents scientifiques de niveau recherche, publiés ou non, émanant des établissements d'enseignement et de recherche français ou étrangers, des laboratoires publics ou privés. 


\section{Microwave phase noise properties of optical links involving small signal and gain saturated optical amplifiers}

\author{
V. Auroux ${ }^{1,2}$, A. Fernandez ${ }^{1,3}$, O. Llopis ${ }^{1}$ \\ ${ }^{1}$ CNRS, LAAS, Univ. de Toulouse, France \\ ${ }^{3}$ Univ. de Toulouse, UPS, LAAS, Toulouse, France \\ 7 avenue du Colonel Roche ; BP 54200 ; 31031 Toulouse, \\ France
}

\author{
P.H. Merrer ${ }^{2}$, A. Vouzellaud ${ }^{2}$ \\ ${ }^{2}$ OSAT \\ 16 avenue Didier Daurat, Toulouse, France
}

\begin{abstract}
Phase noise of optical links involving optical amplifiers is investigated. The amplifier appears to be a significant contributor to the link phase noise and this paper aims at determining in which conditions the optical amplifier is noiseless. A comparison between Erbium Doped Fiber Amplifiers and Booster Optical Amplifiers is drawn. The evolution of noise floor is finally related to noise figure measurements.
\end{abstract}

Keywords-phase noise; optical amplifiers; EDFA; SOA; BOA; noise figure; optical links; microwave optics

\section{INTRODUCTION}

Noiseless optical links have widespread applications in the telecommunications field, such as optical distribution of a frequency standard [1] or high spectral purity signals distribution in satellite [2]. In some cases, the signal received with a classical optical link (laser + modulator + photodiode) is weak and the result is a strong degradation of the signal to noise ratio. This is the case when a clock distribution is performed on a large number of receivers, or on a long distance. In these cases, the use of an optical amplifier can help in retrieving a high signal level on the photodetector. Moreover, the optical amplifier is an essential component of some high quality frequency sources, such as coupled optoelectronic oscillators [3] in which a mode locked laser is coupled to a microwave oscillation. In this specific case, high gain and strong saturation power helps to design RF amplifier-free devices [4]. However, when an optical amplifier is involved in an optical link, or a microwaveoptical system, it adds its own noise components, and it can be the main contributor to phase noise both close and far away from the carrier frequency.

It is thus essential to investigate on the possible techniques to reduce this noise contribution, which include the choice of the optical amplifier and the choice of its operating conditions. Several types of optical amplifiers technologies are today commercially available. In this paper, we will focus on two amplifiers: an Erbium Doped Fiber Amplifier (EDFA) and a Booster Optical Amplifier (BOA), which is a high power Semiconductor Optical Amplifier (SOA). These devices are generally described through their small signal gain, saturation output power and noise figure, and we have investigated their phase noise performance in relation with these parameters.
Phase noise performances in optical links can be degraded by optical amplitude to RF phase conversion on photodiode. Specific studies have been carried out to avoid this conversion [4]. Thus, in our experiments, the optical power on the photodiode has been controlled to minimize this conversion factor.

Finally, a theoretical approach is presented to predict the evolution of the phase noise floors regarding to the variations of the amplifier noise figure and gain. It enables to determine the carrier to noise ratio (CNR) which corresponds to the phase noise floor at the photodiode output.

\section{PHASE NOISE IN OPTICAL LINKS}

\section{A. Measurement setup}

The investigations have been performed on an optical link using sucessively two different optical amplifiers, the other elements of the link being unchanged. The microwave residual phase noise of the link has been characterized with the measurement setup detailed in [5] and depicted in Fig. 1. A $3.5 \mathrm{GHz}$ low phase noise and low amplitude noise dielectric resonator oscillator (DRO), together with and a cross correlation phase detection technique, have been used. A delay on the RF path has also been adjusted to correspond to the delay introduced by the optical link, in order to cancel a possible parasitic detection of the DRO phase noise. The microwave phase noise added to the optical link has been measured in saturated and unsaturated conditions for both amplifiers.

\section{B. Amplitude to phase noise conversion}

The first phase noise results have revealed an important phase noise level close to the carrier (below $1 \mathrm{kHz}$ ) for the EDFA. Hence, to determine the source of this noise, we have measured the amplitude noise of this amplifier at baseband.

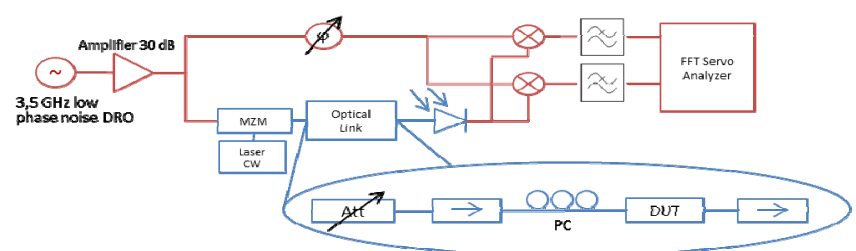

Figure 1 : Residual phase noise measurement setup (Att : variable attenuator, MZM : Mach Zehnder Modulator, PC : polarization controller) 


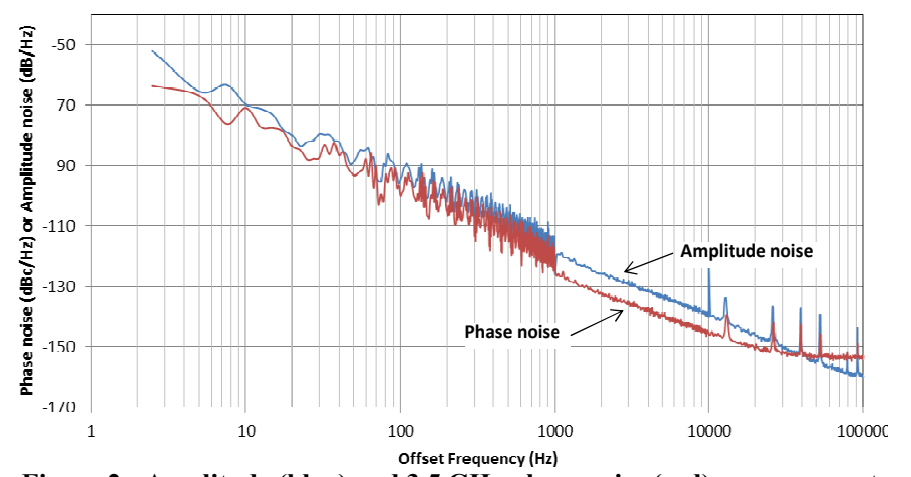

Figure 2 : Amplitude (blue) and 3.5 GHz phase noise (red) measurement for an EDFA

The low frequency amplitude noise and the microwave phase noise of this EDFA are displayed together on Fig. 2. The shape of both spectra is very similar (with the exception of the noise floor which is different at RF). We can thus conclude that amplitude to phase conversion is responsible for the bump observed on the $3.5 \mathrm{GHz}$ phase noise plot close to the carrier.

A previous study had been carried out to minimize the amplitude to phase conversion on the photodiode [6], based on the technique described in [4]. It consists in adjusting the optical power for which the photodiode has no RF phase fluctuations regarding to optical power fluctuations. In our case, this specific optical power level has been measured to be close to $10 \mathrm{~mW}$. Therefore, in all the measurements presented hereafter, the photodiode is used with $10 \mathrm{~mW}$ optical power. The effect of such an optimization is depicted in Figure 3: the optical link with the EDFA is measured with an attenuator inserted between the amplifier and the photodiode, and the phase noise is plotted in two different power conditions $(10 \mathrm{~mW}$ and $16 \mathrm{~mW})$. An improvement in phase noise up to $15 \mathrm{~dB}$ has been observed at the zero phase slope of the photodiode. This method was however not efficient with the BOA. The RF phase fluctuations in this amplifier may result from a different process than simple amplitude to phase conversion at the photodiode level. However, also for the EDFA, the phase noise is not totally suppressed by optimizing the amplitude to phase conversion at the photodiode level.

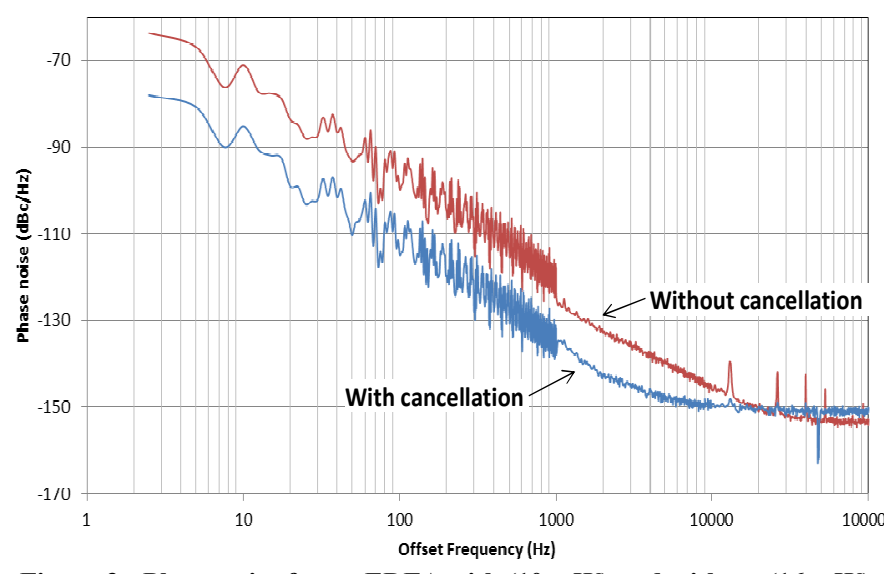

Figure 3 : Phase noise for an EDFA with $(10 \mathrm{~mW})$ and without $(16 \mathrm{~mW})$ cancellation of amplitude to phase conversion

\section{EDFA VS BOA PERFORMANCES}

Operating conditions of an optical amplifier can affect phase noise performances of the optical link. The aim of this part of the study is to determine in which condition the amplifier is noiseless. Therefore, each amplifier has been characterized in two regimes: a linear regime, where the gain corresponds to the small signal gain, and a saturated regime.

\section{A. Optical amplifier-free noise floor}

To distinguish the phase noise which is added by the amplifier itself, it is necessary to eliminate the phase noise contributions from the other optical link's components. The residual phase noise of the optical link without the amplifier has thus been measured with the same technique. This measurement represents the experiment noise floor, and is reported on both Fig. 4 and Fig. 5. One can observe a phase noise floor of $-155 \mathrm{dBc} / \mathrm{Hz}$ above $10 \mathrm{kHz}$ from the carrier. This phase noise level corresponds to the RIN of the laser we used. Besides, the slope corresponding to the measurement below $1 \mathrm{kHz}$ is higher than $1 / \mathrm{f}$.

\section{B. Amplifiers phase noise}

In order to be able to compare both amplifiers, they are biased (BOA) or pumped (EDFA) in order to get the same small signal gain. Firstly, to distinguish the phase noise which is added by the amplifier itself from the other noise sources, it is necessary to eliminate any other contributions. Measurements for linear and saturated regimes for the EDFA and the BOA are reported on Fig. 4 and Fig 5.

On the one hand, the EDFA presents the same close to the carrier phase noise level whether it is saturated or not whereas the BOA phase noise is reduced in saturated regime. One can notice that the BOA phase noise close to the carrier is lower than the EDFA phase noise, with an improvement of more than $10 \mathrm{~dB}$ at $10 \mathrm{~Hz}$. On the other hand, the BOA and the EDFA have roughly the same behavior towards phase noise floors. Both amplifiers present an important reduction of their noise floors in the saturated regime. This is mainly due to the decrease of the gain. This noise contribution is additive noise: as we reduced the CNR by saturating the amplifier, the noise floor decreased. A reduction up to $15 \mathrm{~dB}$ has been observed for the EDFA and close to $10 \mathrm{~dB}$ for the

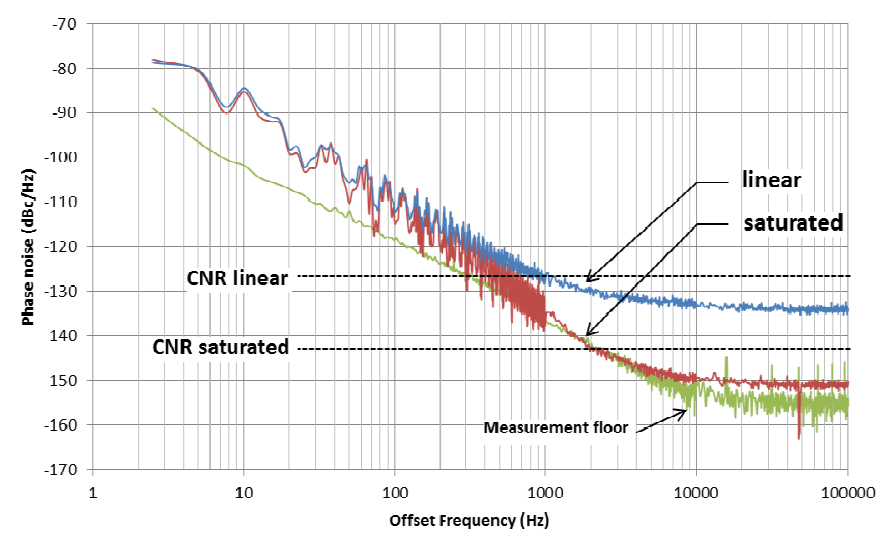

Figure 4 : Phase noise of EDFA in saturated (red) and linear (blue) regimes 


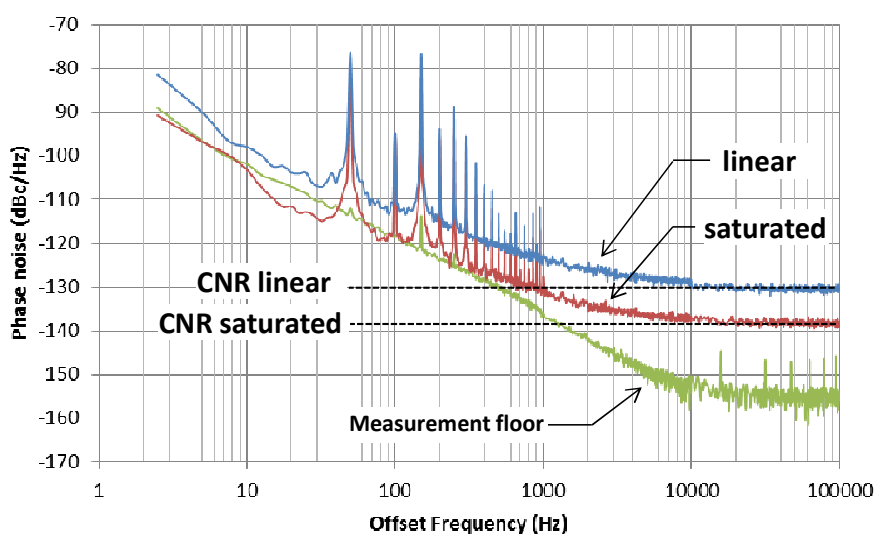

Figure 5 : Phase noise of BOA in saturated (red) and linear (blue) regimes

BOA. Besides, EDFA's noise floors are lower than BOA's, with more than $10 \mathrm{~dB}$ improvement at $100 \mathrm{kHz}$. This could be explained by the difference of noise figure between the two amplifiers. The evolution of the noise floors can be correlated to the variation of the noise figures and gain of the amplifiers. This behavior is detailed in the following section.

Concerning the comparison between EDFA and BOA for time and frequency applications, there is a tradeoff between their respective performances whether one looks for close to the carrier or far from carrier performance.

\section{Noise floors evolution}

There are several techniques to measure the noise figure of an optical amplifier. We have used the optical way to measure this parameter, as detailed in [8]. The evolution of the noise figure with respect to the input power has been measured for both amplifiers. The noise figure can be expressed as follow:

$$
F=\frac{2 P_{A S E}}{G \cdot h v \cdot \Delta v}
$$

where $\mathrm{P}_{\mathrm{ASE}}$ is the average power of spontaneous emission integrated on the optical spectral analyzer's resolution bandwidth $\Delta v$ and $\mathrm{G}$ is the amplifier gain.

Then, it is necessary to express the amplifier phase noise density on the photodiode. This phase noise floor can be related to the optical $\mathrm{CNR}$, which depends on the noise figure, the photodiode current $\mathrm{I}_{\mathrm{PD}}$ and the optical input power $\mathrm{P}_{\text {in }}$ as follow [8]:

$$
S_{\phi}=\frac{2 h v \cdot I_{P D}^{2} \cdot F}{P_{\text {in }}}
$$

From this equation, we can finally express the carrier to noise ratio for the optical link:

$$
C N R=10 \log \left(\frac{I_{P D}{ }^{2} m^{2}}{2\left(R I N \cdot I_{P D}{ }^{2}+\frac{4 k T}{R_{d}}+2 q I_{P D}+\frac{2 h v \cdot I_{P D}{ }^{2} \cdot F}{P_{\text {in }}}\right)}\right)
$$

where RIN stands for the relative intensity noise of the laser, $\mathrm{m}$ the modulation depth, $4 \mathrm{kT} / \mathrm{R}_{\mathrm{d}}$ and $2 \mathrm{qI}_{\mathrm{PD}}$ represent respectively the thermal noise and the shot noise on the photodiode.

Using equation (3), we can compute the noise floor of the measurements described in previous section. Those CNR floors are reported (dashed lines) for each measurement in Fig. 4 and Fig. 5. We can observe that for the BOA, both measurements floors match the calculated CNR associated. It confirms that the major phase noise contribution comes from the amplifier. Indeed, the ratio $\mathrm{F} / \mathrm{P}_{\text {in }}$ decreases as we saturate the amplifier since the noise figure is stable in linear regime and slightly increases with the saturation. However, results for the EDFA do not fit the measurements. As one can see on Fig. 4, CNR floors are several $\mathrm{dB}$ higher than measured noise floors. Yet, the variation of noise floors with respect to noise figure and gain evolution is well predicted.

\section{CONCLUSION}

Microwave phase noise performances of optical links including two types of optical amplifiers, an EDFA and a BOA, has been presented. A correlation between amplitude noise and phase noise close to the carrier for the EDFA has been shown. It led us to adjust the optical power on the photodiode to minimize the amplitude to phase conversion. Then, a study of saturation state for both amplifiers has been carried out to determine the optimal operating conditions. It has been highlighted that there is a tradeoff between EDFA and BOA whether one seeks for an optimization of close to carrier performance or of the far from carrier noise floors. The BOA presents a low $1 / \mathrm{f}$ phase noise close to the carrier while the EDFA features a better noise floor due to its lower noise figure. Finally, a model has been presented to predict the variation of the noise floors for both amplifiers.

\section{REFERENCES}

[1] F. Narbonneau, M. Lours, S. Bize, A. Clairon, G. Santarelli, O. Lopez, C. Daussy, A. Amy-Klein, et C. Chardonnet, «High resolution frequency standard dissemination via optical fiber metropolitan network », Rev. Sci. Instrum., vol. 77, nº 6, p. 064701 , 2006.

[2] B. Onillon, B. Bénazet, et O. Llopis, « Advanced microwave optical links for LO distribution in satellite payloads », in Microwave Photonics, 2006. MWP'06. International Topical Meeting on, 2006, p. 1-4.

[3] X. S. Yao, L. Davis, et L. Maleki, «Coupled optoelectronic oscillators for generating both RF signal and optical pulses », $J$. Light. Technol., vol. 18, $\mathrm{n}^{\mathrm{O}}$ 1, p. 73, 2000.

[4] W. Loh, S. Yegnanarayanan, J. J. Plant, F. J. O’Donnell, M. E. Grein, J. Klamkin, S. M. Duff, et P. W. Juodawlkis, « Low-noise RFamplifier-free slab-coupled optical waveguide coupled optoelectronic oscillators: physics and operation ", Opt. Express, vol. 20, $\mathrm{n}^{\circ}$ 17, p. 19420-19430, 2012.

[5] D. Eliyahu, D. Seidel, et L. Maleki, « RF Amplitude and Phase-Noise Reduction of an Optical Link and an Opto-Electronic Oscillator », IEEE Trans. Microw. Theory Tech., vol. 56, n 2, p. 449-456, 2008.

[6] G. Cibiel, M. Régis, E. Tournier, et O. Llopis, « AM noise impact on low level phase noise measurements », Ultrason. Ferroelectr. Freq. Control IEEE Trans. On, vol. 49, nº 6, p. 784-788, 2002.

[7] Z. Abdallah, A. Rumeau, J. Maxin, A. Fernandez, L. Morvan, O. Llopis, et G. Cibiel, « Photodiode nonlinear modeling and its impact on optical links phase noise », EFTF proceeding, 2014.

[8] D. M. Baney, P. Gallion, et R. S. Tucker, « Theory and measurement techniques for the noise figure of optical amplifiers », Opt. Fiber Technol., vol. 6, $\mathrm{n}^{\mathrm{o}} 2$, p. 122-154, 2000. 